Sharif University of Technology
Scientia Iranica
SCIENTIA

\title{
Experimental estimation heat flux and heat transfer coefficient by using inverse methods
}

\author{
S.D. Farahani*, A.R. Najafi, F. Kowsary and M. Ashjaee \\ Department of Mechanical Engineering, University College of Engineering, University of Tehran, Tehran, P.O. Box 11155-4563,
} Iran.

Received 1 December 2014; received in revised form 27 July 2015; accepted 15 September 2015

\author{
KEYWORDS \\ Inverse method; \\ Heat flux; \\ Convective heat \\ transfer coefficient; \\ Adjoint equation.
}

\begin{abstract}
The purpose of this paper is to present experimental applications of the inverse heat transfer methods (conjugate gradient method and sequential method). Three experiments are designed to estimate the heat flux and the heat transfer coefficients. In the third experiment, convective heat transfer coefficient is estimated directly and indirectly. In direct estimation, the conjugate gradient method with adjoint equation is used. The results show that inverse heat transfer methods are able to estimate the desired parameters with good accuracy in experimental state when mathematical model and boundary condition are correct and appropriate with experimental model.

(C) 2016 Sharif University of Technology. All rights reserved.
\end{abstract}

\section{Introduction}

Being highly sensitive to random errors (noise) that inherently exist in measured temperature data, inverse heat conduction problems are mathematically illposed. In order to alleviate this problem, regularization techniques are utilized [1]. Su et al. [2] used inverse process method combined with grey prediction model to estimate the inner surface geometry of a cylindrical furnace wall. Inverse methods are commonly used for thermo-physical parameter estimation problems. Beck [3] estimated the thermal conductivity simultaneously with the volumetric heat capacity of nickel by one-dimensional transient temperature measurements. The research done by Jurkowski and Jarny [4] and Garnier et al. [5] showed that small sensitivity coefficients or the unbalance of the sensitivity matrix result in instability of the estimation procedure. This particular remark goes along with the fact that both

\footnotetext{
* Corresponding author. Tel.: +982161114024; Fax: +982188013029 E-mail address: sdavoodabadifarahani@gmail.com (S.D. Farahani)
}

the Gauss and modified Box-Kanemasu methods [6] have shown that the resulting instabilities cause the divergence of the method when used with models that contain correlated or nearly correlated thermal properties. Several different approaches have been used to address this problem. One approach is to modify the experimental design. Nevertheless, modifications of the experimental design, such as the use of internal sensors, are not always feasible, especially when nondestructive testing is required. In addition, the use of embedded thermocouples can be a source of important bias. Taler [7] compared two techniquesSingular Value Decomposition (SVD) and LevenbergMarquardt - used in determining the space variable heat transfer coefficient on a tube circumference. Dong et al. proposed a method of fundamental solutions for inverse heat conduction problems in an anisotropic medium [8]. Another approach is applying regularization methods, which can be employed in two ways. Although the regularization methods introduce a bias into the estimation, they significantly stabilize the solution. In general, methods of solving the inverse heat conduction problem can be divided into two main groups: sequential methods and the whole- 
domain methods [9]. Each of the groups has its own advantages. Sequential methods can be used for real-time estimation and require less memory and computational time. Whole-domain methods, on the other hand, are more accurate than the sequential methods since the whole-domain methods use all the measured temperatures simultaneously in estimation of any unknown parameter or function. The wellknown whole-domain methods are the conjugate gradient method and the Tikhonov regularization method. One type of regularization, known as the "Tikhonov Regularization," adds a penalty term to the objective function. Additional comments on this type of regularization can be found in chapter 2 of the book by Woodbury [10]. The conjugate gradient method has been widely used in the literature and is known as one of the successful algorithms of IHCP, especially for problems of which the boundary conditions cover the major part of the boundary [11]. Kowsary and Farahani [12] applied the de-noised measurement data by using mollification method before the standard IHCP algorithm for estimated heat flux for classical inverse problems. The filter method [13] used in this paper is a representation of one of many IHCP solution methods, such as Tikhonov Regularization, in a digital filter form.

In most of the previous studies on inverse heat transfer, measured temperatures for the inverse method are generated by using numerical simulations and by adding random errors. In this study, three experiments are designed that investigate ability of the standard methods of inverse heat transfer. The designed experiments include estimation of heat flux and free convective heat transfer coefficients. Metal plates are made of stainless steel (AISI- 304) with 250, 70, and $5 \mathrm{~mm}$ of length, width, and thickness, respectively. K-type (TP01) thermocouple is used to measure temperature. Temperatures are recorded by using a data acquisition system with time. Errors in experimental data can be classified into two categories: fixed and random. The fixed errors have the same magnitude in each measurement while random errors have different magnitudes in each experiment. Although the fixed errors may be removed by appropriate calibration, the random errors cannot be removed. The accuracy of the K-type thermocouple is $0.1^{\circ} \mathrm{C}$. It is assumed that the uncertainty of the physical properties and geometry is 0.01 .

Measured temperatures before applying the inverse heat transfer method are de-noised by using a moving average filter. The moving average is the most common filter, mainly because it is the easiest digital filter to understand and use. In spite of its simplicity, the moving average filter is optimal for reducing random noise of signal. Two inverse methods, sequential specification function method [8] and conjugate gradient method [11], are used in this paper.

\subsection{First experiment}

The first case study is performed on a rectangular body as shown in Figure 1(a). The geometry and boundary conditions of the first test case are presented as well as the locations of the sensors. Two plates have the same boundary conditions; thus, one plate is modeled. Assuming constant thermal properties, the heat equation is written as:

$$
\begin{aligned}
& \frac{\partial^{2} T}{\partial x^{2}}=\frac{1}{\alpha} \frac{\partial T}{\partial t} \\
& -\left.k \frac{\partial T}{\partial x}\right|_{x=0}=q(t)(\text { is unknown })-\left.k \frac{\partial T}{\partial x}\right|_{x=E} \\
& =h\left(T-T_{\infty}\right) \\
& \left.T\right|_{t=0}=T_{0} .
\end{aligned}
$$

\subsection{Second experiment}

Geometry and boundary conditions of the second test case are shown in Figure 1(b), and location and

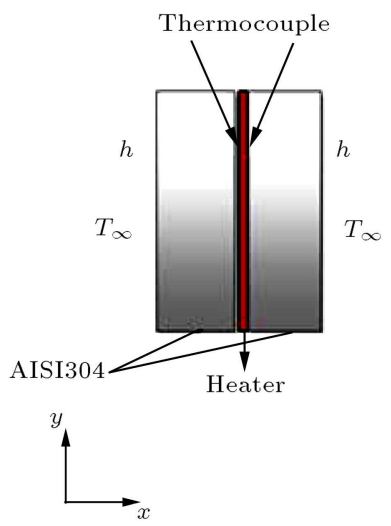

(a)

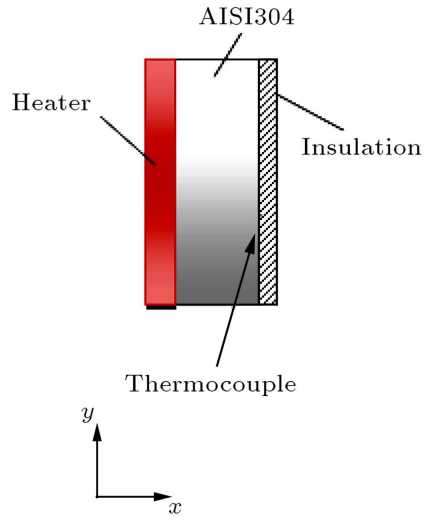

(b)

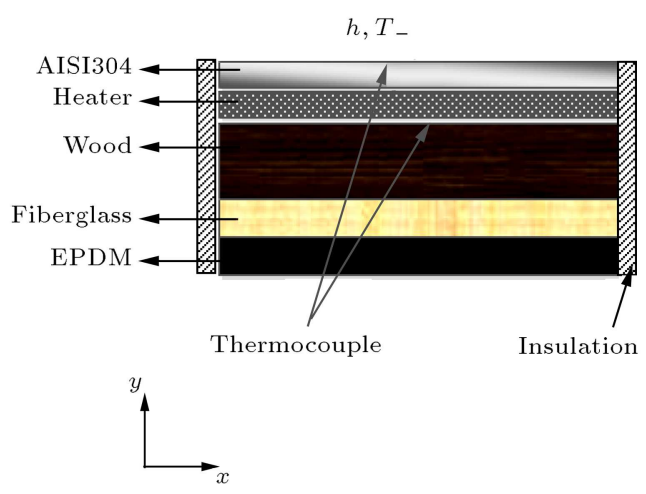

(c)

Figure 1. Schematic of experiments set-up: (a) Fist experiment; (b) the second experiment; and c) the third experiment. 
arrangement of the sensors are shown in Figure 1(b). Assuming constant thermal properties, the governing equation is written as:

$$
\begin{aligned}
& \frac{\partial^{2} T}{\partial y^{2}}=\frac{1}{\alpha} \frac{\partial T}{\partial t} \\
& -\left.k \frac{\partial T}{\partial y}\right|_{x=E}=q(?) ; \quad-\left.k \frac{\partial T}{\partial y}\right|_{x=0}=0
\end{aligned}
$$

$$
\text { and }\left.T\right|_{t=0}=T_{0} \text {. }
$$

\subsection{Third experiment}

At this step, the test is designed to estimate free convection heat transfer coefficient of air by using inverse heat transfer method. Figure 1(c) shows geometry, boundary conditions, and location, and arrangement of the sensors for the third test case. By measuring the temperature of the end of the last layer of insulation, it has been seen that the difference between its temperature and the ambient temperature is little. Thus, the heat loss from the insulation to environment is almost zero, but the amount of heat stored in the first layer of insulation is remarkable. Therefore, the estimation of heat transfer coefficient is done in two steps. First, heat flux transferred from the heater to the insulation is estimated. Then, the heat flux transferred from heater to plate is determined and the heat transfer coefficient is estimated. In inverse methods, mathematical model is needed; thus, the problem should be modeled first. Assuming constant thermal properties, the governing equation of insulation layer is written as:

$$
\begin{aligned}
& \frac{\partial^{2} T}{\partial y^{2}}=\frac{1}{\alpha_{\mathrm{ins}, i}} \frac{\partial T}{\partial t}, \quad i=1,2,3, \\
& -\left.k_{\mathrm{ins}, 1} \frac{\partial T}{\partial y}\right|_{y=0_{1}}=\text { heat loss; } \\
& -\left.k_{\mathrm{ins}, 3} \frac{\partial T}{\partial y}\right|_{y=E_{\mathrm{ins}, i}} \cong 0 \quad \text { and }\left.\quad T\right|_{t=0}=T_{0} .
\end{aligned}
$$

Assuming constant thermal properties, the governing equation of metal plate is written as:

$$
\begin{aligned}
& \frac{\partial^{2} T}{\partial y^{2}}=\frac{1}{\alpha} \frac{\partial T}{\partial t}, \\
& -\left.k \frac{\partial T}{\partial y}\right|_{y=0}=Q_{s}-\text { heat loss; } \\
& -\left.k \frac{\partial T}{\partial y}\right|_{y=E}=q=h\left(T-T_{\infty}\right)+\varepsilon \sigma\left(T^{4}-T_{\infty}^{4}\right), \\
& \left.T\right|_{t=0}=T_{0} .
\end{aligned}
$$

\section{The Conjugate Gradients Method (CGM)}

In order to estimate the heat flux by using the conjugate gradient method, the error function $S$ is also defined as:

$$
S(q)=\sum_{i=1}^{N_{s}} \sum_{m=1}^{N_{m}}\left(T_{i}\left(t_{m}\right)-Y_{i}\left(t_{m}\right)\right)^{2},
$$

where $Y$ is measured temperature at sensor location, and $T$ is the estimated value at sensor location. In heat equation, the directional derivative of $S$ can be used to define the gradient function of $\nabla S$ with respect to $Q$ as follows:

$$
\vec{\nabla} S=-2[X]^{T}([Y]-[T]),
$$

where all the mentioned parameters are evaluated at the sensor location. The sensitivity coefficients with respect to each component of $\vec{q}$ are defined as:

$$
\begin{aligned}
& X_{p}\left(x_{j}, y_{j}, t_{m}\right)=\frac{\partial T\left(x_{j}, y_{j}, t_{m}\right)}{\partial q^{m}} \\
& j=1,2, \cdots, J, \quad m=1,2, \cdots, n .
\end{aligned}
$$

Using the above equation, the conjugate direction $(d)$ can be calculated as:

$$
d^{k}=\nabla S\left(Q^{k}\right)+\gamma^{k} d^{k-1} \text {. }
$$

The conjugate coefficient $\gamma$ is calculated as:

$$
\gamma^{k}=\left(\int_{t=0}^{t_{f}}\left\{\nabla S\left(Q^{k}\right)\right\}^{2} d t\right) /\left(\int_{t=0}^{t_{f}}\left\{\nabla S\left(Q^{k-1}\right)\right\}^{2} d t\right),
$$

where $\gamma^{0}=0$. If $Q^{\prime}=Q^{k}+d^{k}$ is substituted in heat equation, then equation $\Delta T$ will be calculated at the sensor location as follows:

$$
\Delta T=T\left(Q^{\prime}\right)-T\left(Q^{k}\right) .
$$

Therefore, the search step size $(\beta)$ can be obtained as:

$$
\begin{aligned}
\beta^{k}= & \left(\sum_{j=1}^{J} \sum_{m=1}^{M}\left(T_{j}\left(t_{m}\right)-Y_{j}^{m}\left(t_{m}\right)\right) \Delta T_{j}\left(t_{m}\right)\right) \\
& /\left(\sum_{j=1}^{k} \sum_{m=1}^{M} \Delta T_{j}^{2}\left(t_{m}\right)\right) .
\end{aligned}
$$

In this method, an iterative procedure is used to estimate the imposed heat flux. This iterative method can be summarized by the following equation:

$$
Q^{k+1}=Q^{k}-\beta^{k} d^{k}
$$

where " $d$ " is a conjugate direction and $\beta$ is the search step size. The computational procedure for the solution of this inverse problem may be summarized as follows: Suppose $Q^{n}$ is available at iteration $n$. 
- Step 1. Solve the direct problem for $T$;

- Step 2. Examine the stopping criterion considering $S(Q)<\dot{\alpha}$, where $\alpha$ is a small specified number. Continue if not satisfied.

The "Discrepancy Principle" in the conjugate gradient method has been used in this study. In this method, the iterations are terminated prematurely when the following criterion is satisfied [11]:

$$
S(Q)<\dot{\alpha} .
$$

In this case, the iterations stop when the residuals between measured and estimated temperatures are of the same order of magnitude of the measurement errors. That is:

$$
|Y(t)-T(X, t)|<\sigma \quad \text { (i.e. standard deviation). }
$$

- Step 3. Solve the sensitivity equation for $X$;

- Step 4. Compute the gradient of the functional $\nabla S$ from Eq. (6);

- Step 5. Compute the conjugate coefficient $\gamma^{k}$ and direction of descent $d^{k}$ from Eqs. (8) and (9), respectively;

- Step 6. Set $\Delta Q=d^{k}$ in heat equation for the problem and solve it for the calculated $\Delta T$;

- Step 7. Compute the search step size $\beta^{k}$ by Eq. (11);

- Step 8. Compute the new estimation for $Q^{n+1}$ from Eq. (12) and return to Step 1.

\section{The Sequential Function Specification Method (SFSM)}

This inverse method is sequential and uses Beck's function specification approach, where heat fluxes of $r$ "future" time steps are temporarily assumed constant and used to add stability to the estimations. It is assumed that heat fluxes from times $1,2, \cdots,(m-1)$ are estimated, and now the unknowns in time $m$ are to be evaluated. The standard form of the IHCP is the matrix equation (see [9]):

$$
T=\left.T\right|_{q=0}+X q,
$$

where $\left.T\right|_{q=0}$ is the calculated temperatures at sensor locations from the solution of the direct problem using $q_{1}, \cdots, q_{m-1}$ and setting Eq. (5) to zero. For $N p$ heat flux parameters, $N s$ temperature sensors, and $r$ future times:

$$
T=\left[\begin{array}{c}
T(m) \\
T(m+1) \\
\vdots \\
T(m+r-1)
\end{array}\right] \text { and } \quad T(i)=\left[\begin{array}{c}
T_{1}(i) \\
T_{2}(i) \\
\vdots \\
T_{N s}(i)
\end{array}\right] \text { (16) }
$$

where $T$ is an $N s . r \times 1$ matrix:

$$
q=\left[\begin{array}{c}
q(m) \\
q(m+1) \\
\vdots \\
q(m+r-1)
\end{array}\right] \quad \text { and } \quad q(i)=\left[\begin{array}{c}
q_{1}(i) \\
q_{2}(i) \\
\vdots \\
q_{N p}(i)
\end{array}\right]
$$

where $T$ is an $N p . r \times 1$ matrix:

$$
Z=\left[\begin{array}{cccc}
a(1) & & & \\
a(2) & a(1) & & \\
\vdots & \vdots & \ddots & \\
a(r) & a(r-1) & \cdots & a(1)
\end{array}\right]
$$

where $Z$ is an $N$ s. $\times N$ p.r matrix; and:

$$
\begin{aligned}
a & =\left[\begin{array}{cccc}
a_{11}(i) & a_{12}(i) & \cdots & a_{1 N p}(i) \\
a_{21}(i) & a_{22}(i) & \cdots & a_{2 N p}(i) \\
\vdots & \vdots & & \vdots \\
a_{N s 1}(i) & a_{N s 2}(i) & \cdots & a_{N s N p}(i)
\end{array}\right], \\
a_{j p} & =\frac{\partial T\left(x_{j}, t_{i}\right)}{\partial q_{p}}
\end{aligned}
$$

where $a(i)$ is a matrix of $N s \times N p$.

Note that there are $N p$ unknown heat flux components at each time $t_{m}$. There are $N s$ measurements at that time, and $N s$ should not be less than $N p$. To produce stable results, we use $r$ matrix equations in a least-squares method. The sum of squares of the difference between calculated and measured temperatures, in matrix form, is:

$$
S=(Y-T)^{t}(Y-T) .
$$

With the temporary assumption of:

$$
\begin{aligned}
q_{1}(m) & =q_{1}(m+1)=\cdots \\
& =q_{1}(m+r-1) \cdots q_{N p}(m)=q_{N p}(m+1) \\
& =\cdots=q_{N p}(m+r-1),
\end{aligned}
$$

here, the function to be minimized is:

$$
S=\left(Y-\left.T\right|_{q=0}-Z q\right)^{T}\left(Y-\left.T\right|_{q=0}-Z q\right),
$$

where:

$$
Z=X A, \quad A=\left[\begin{array}{c}
A(1) \\
\vdots \\
A(r)
\end{array}\right]
$$

For a constant $q$ assumption, $A(i)=I_{N p \times N p}$ where $I$ is unity matrix. 
The matrix derivative of Eq. (22) with respect to $q$ (in order to minimize $S$ ) yields the estimator equation:

$$
\hat{q}=\left(Z^{T} Z\right)^{-1} Z^{T}(Y-T \mid q=0)
$$

which gives the $\hat{q}(m)$ vector as is defined by Eq. (17). The following estimation procedure is employed: $X$ and, consequently, $Z$ are calculated; $m$ is set to one. Estimation begins with the calculation of $\left.T\right|_{q=0}$ using the direct problem and subsequent application of Eq. (24). Then, $m$ is increased by one, $\left.T\right|_{q=0}$ is recalculated, and the estimator equation is used again.

\section{Moving average filter}

A moving average filter smoothes data by replacing each data point with the average of the neighboring data points defined within the span. This process is equivalent to low-pass filtering with the response of the smoothing given by the difference equation:

$$
\begin{aligned}
Y_{s}(i)= & \frac{1}{2 N+1}(Y(i+N)+Y(i+N-1) \\
& +\cdots+Y(i-N)),
\end{aligned}
$$

where $Y(i)$ is noisy data and $Y_{s}(i)$ is the smoothed value for the $i$ th data point, $N$ is the number of neighboring data points on either side of $Y_{s}(i)$, and $2 N+1$ is the span. The moving average smoothing method used by curve fitting follows these rules:

1. The span must be odd;

2. The data point to be smoothed must be at the center of the span;

3. The span is adjusted for data points that cannot accommodate the specified the specified number of neighbors on either side;

4. The end points are not smoothed because a span cannot be defined.

\section{Result and discussion}

Sequential method and conjugate gradient method were used in this study. The goal of the designed experiments is estimation of time history for the unknown parameter by using measured temperatures in experimental state. Time step for recorded temperatures is 0.1 second. For solving, inverse technique is used in 0.1 second. Finite Element Method is used in numerical solutions where ANSYS capabilities are utilized in the mesh generation and numerical solution of the problem in order to evade the need for coding the direct heat conduction problem. By simulating the problem in the graphic user interface of the software and saving it in the form of a function, the numerical solution of the problem can be performed merely by calling the saved function. Inverse algorithms are written in ANSYS Parametric Design Language (APDL). The element type is Plane-55. In the following, processes of solving three tests will be discussed. In the first test, before using inverse algorithm, sensitivity coefficients are calculated. Governing equations for pulse sensitivity coefficients are obtained by taking the derivative of the heat (Eq. (1)) with respect to each $q$, which yields:

$$
\begin{aligned}
& \frac{\partial^{2} X}{\partial x^{2}}=\frac{1}{\alpha} \frac{\partial X}{\partial t} \\
& -\left.k \frac{\partial X}{\partial x}\right|_{x=E}=h X, \\
& -\left.k \frac{\partial X}{\partial x}\right|_{x=0}= \begin{cases}1 x_{n}<x<x_{n+1} & \text { otherwise } \\
0 & \end{cases} \\
& \left.X\right|_{t=0}=0
\end{aligned}
$$

Sensor is located in active surface (see Figure 1(a)). Now, unknown heat flux is estimated by using the described equations, measured temperatures, and inverse method. Stop criterion for conjugate gradient method is $10^{-6}$ and regularization parameter for sequential specification method is 5 . Figure 2 shows the results of using inverse technique. Heat fluxes of two plates are estimated with good accuracy. It is noted that the purpose of 'Exact' word in all of the figures is the value of the unknown parameter in experimental model that must be estimated by inverse method. Table 1 shows that the conjugate gradient method estimated heat flux with smaller error than sequential method. In the second test, the governing equations for pulse sensitivity coefficients are obtained by taking

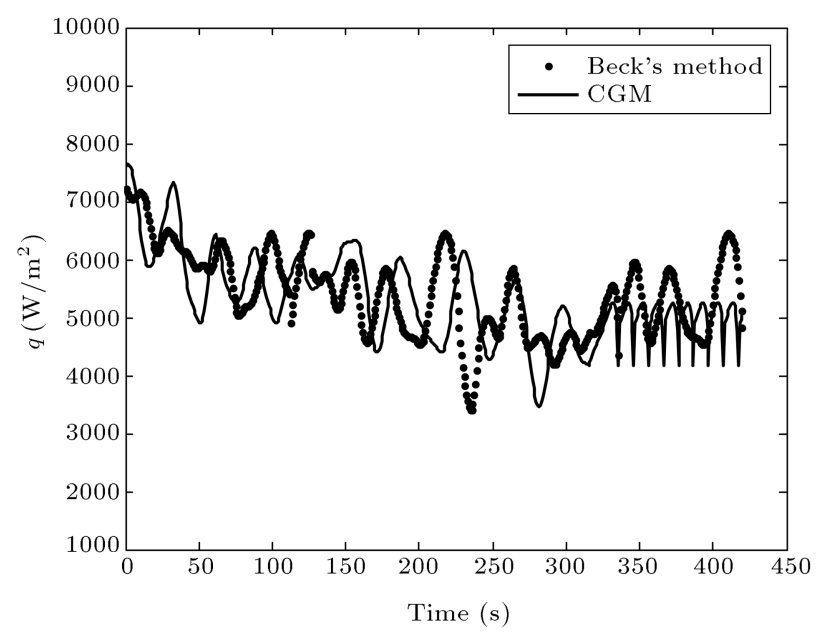

Figure 2. Estimation of heat flux by using Sequential Function Specification Method (SFSM) and using Conjugate Gradient Method (CGM) in the first experiment. 
Table 1. The estimated heat flux and error percent in the first experiment.

\begin{tabular}{lccccc}
\hline & Plate 1 & Plate 2 & $\begin{array}{c}\text { Estimated heater's } \\
\text { heat flux }\end{array}$ & $\begin{array}{c}\text { Exact heater's } \\
\text { heat flux }\end{array}$ & $\begin{array}{c}\text { Error } \\
\text { percent }\end{array}$ \\
\hline Beck's method & 5416.9 & 6408.1 & 10825.4 & 10000.0 & $8.3 \%$ \\
Conjugate gradient method & 5229.1 & 5308.1 & 10537.5 & 10000.0 & $5.4 \%$ \\
\hline
\end{tabular}

the derivative of the heat (Eq. (2)) with respect to each $q$, which yields:

$$
\begin{aligned}
& \frac{\partial^{2} X}{\partial y^{2}}=\frac{1}{\alpha} \frac{\partial X}{\partial t}, \\
& \left.\frac{\partial X}{\partial x}\right|_{x=0}=0, \\
& -\left.k \frac{\partial X}{\partial x}\right|_{x=E}= \begin{cases}1 & x_{n}<x<x_{n+1} \\
0 & \text { otherwise }\end{cases} \\
& \left.X\right|_{t=0}=0 .
\end{aligned}
$$

Sensor is located in inactive surface; thus, sensitivity coefficient value is very small due to the phenomenon of diffusion and time lagging. Now, unknown heat flux is estimated by using the described equations, measured temperatures, and inverse method. In this step, stop criterion for conjugate gradient method is $10^{-6}$ and regularization parameter for sequential specification method is 5. The results show that heat flux is estimated well (Figure 3(a) and (b)). The conjugate gradient method and sequential method are enabled that estimate unknown heat flux in this case. Relative error of estimation for conjugate gradient method is smaller than sequential method (Table 2). In the second section of this test, the plate is exposed to step heat flux. In the previous section, the conjugate gradient method was more accurate than sequential method. Thus, conjugate gradient method is used for estimation step of heat flux. Results of estimation are shown in Figure 4. Error of estimation is almost $10 \%$. The result is acceptable and good as this case study is highly ill-posed. In the last test for estimation of the heat transfer coefficient, two approaches can be used: (a) direct estimation and (b) estimation of $q(x, t)$, subsequent calculation of $T_{\text {surf }}(x, t)$, and then using Newton's law of cooling. While direct estimation might seem more appealing, the second approach causes IHCP to remain linear, thus eliminating the need for iteration, which accelerates the solution considerably.

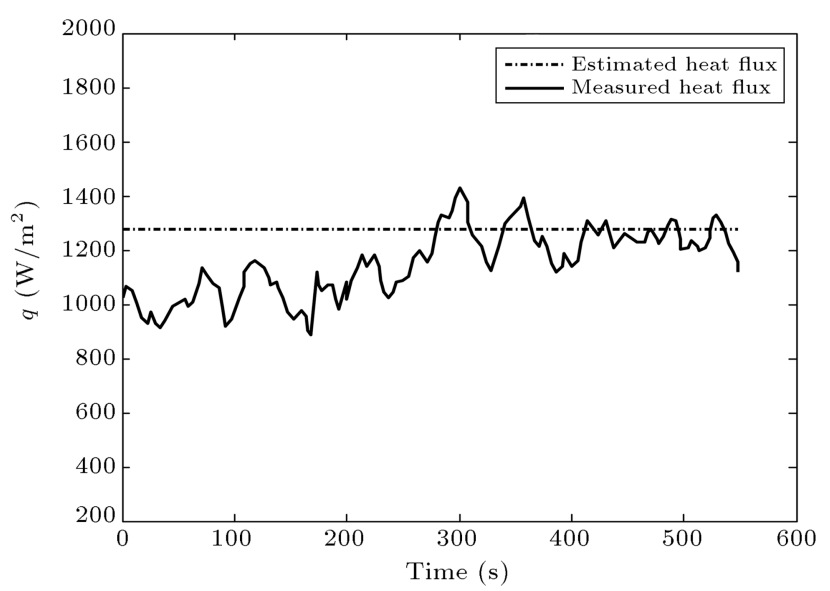

(a)

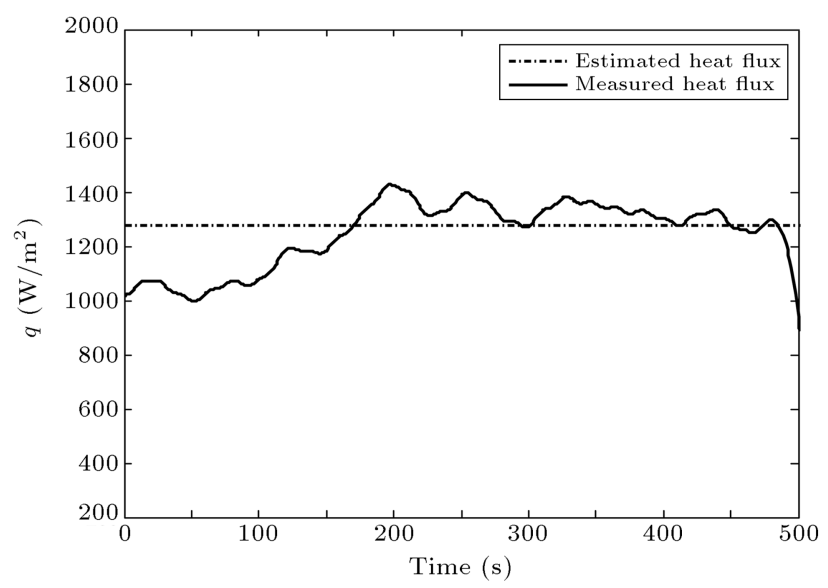

(b)

Figure 3. Estimation of heat flux by using (a) Sequential Function Specification Method (SFSM), and (b) Conjugate Gradient Method (CGM) in the second experiment.

Note that in the present set-up, a heating foil generates a constant known heat flux of $Q_{s}$ on the top surface. A part of $Q_{s}$, namely $q(x, t)$, is conducted in the first layer of insulations estimated by the IHCP and calculated $Q_{c}$. Then, the heat flux $\left(q^{\prime}\right)$ transferred to fluid is estimated by the IHCP (see Figure 5). This value is summation of convection heat transfer and radiation heat transfer. The heat transfer coefficient is then

Table 2. The estimated heat flux and error percent in the second experiment.

\begin{tabular}{lccc}
\hline & $\begin{array}{c}\text { Estimated } \\
\text { heat flux }\end{array}$ & $\begin{array}{c}\text { Exact } \\
\text { heat flux }\end{array}$ & $\begin{array}{c}\text { Error } \\
\text { percent }\end{array}$ \\
\hline Conjugate gradient method & 1232.5 & 1274.300 & $3.26 \%$ \\
Beck's method & 1150.9 & 1274.300 & $9.46 \%$ \\
\hline
\end{tabular}




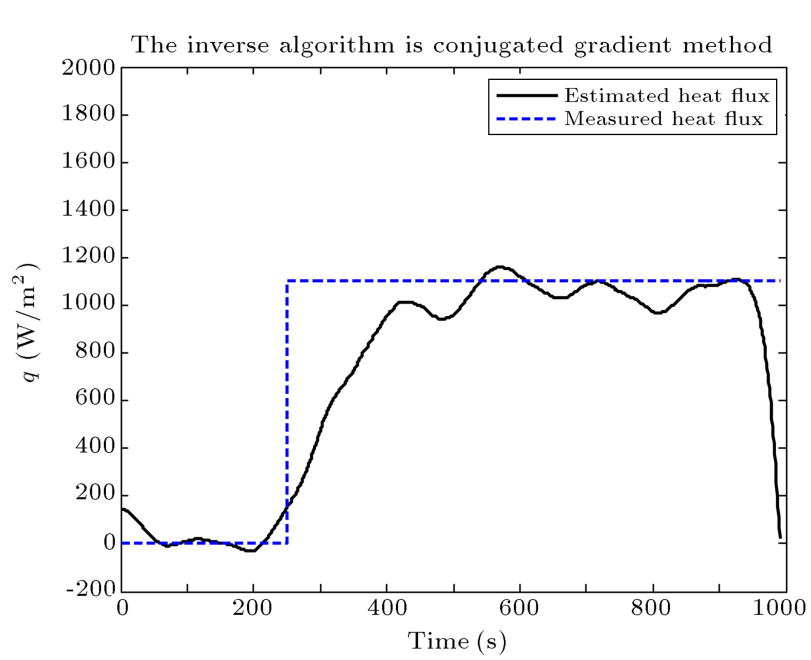

Figure 4. Estimation step of heat flux by using Conjugate Gradient Method (CGM) in the second experiment.

calculated using the remaining part, which is carried out by the jet, using Newton's law of cooling:

$$
h(x)=\frac{q^{\prime}-\varepsilon \sigma\left(T^{4}-T_{\infty}^{4}\right)}{T_{\mathrm{surf}}(x, t)-T_{\infty}},
$$

where $T_{\text {surf }}(x, t)$ and $T_{\infty}$ are the top surface and the environment temperatures, respectively. In continuation, this test includes two steps (see Figure 5). Two sensors are used in this test; one is located in $0.5 \mathrm{~mm}$ from surface that is exposed to air flow and another is located on active surface of insulation. In the first step, the governing equations for pulse sensitivity coefficients are obtained by taking the derivative of the heat (Eq. (3)) with respect to each $q$, which yields:

$$
\begin{aligned}
& \frac{\partial^{2} X}{\partial x^{2}}+\frac{\partial^{2} X}{\partial y^{2}}=\frac{1}{\alpha_{\mathrm{ins}, i}} \frac{\partial X}{\partial t}, \quad i=1,2,3, \\
& \left.\frac{\partial X}{\partial x}\right|_{x=0}=\left.\frac{\partial X}{\partial x}\right|_{x=L}=0
\end{aligned}
$$

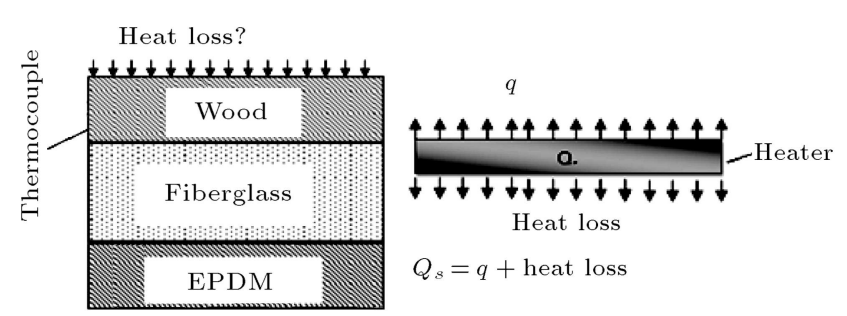

(1)

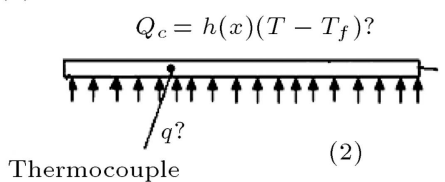

AISI

Figure 5. Schematic of solution process for the third experiment.

$$
\begin{aligned}
& -\left.k_{\mathrm{ins}, 1} \frac{\partial X}{\partial y}\right|_{y=0_{1}}= \begin{cases}1 & x_{n}<x<x_{n+1} \\
0 & \text { otherwise }\end{cases} \\
& -\left.k_{\mathrm{ins}, 3} \frac{\partial X}{\partial y}\right|_{y=E_{\mathrm{ins}, i}} \cong 0 \quad \text { and }\left.X\right|_{t=0}=0 .
\end{aligned}
$$

In this step, stop criterion for conjugate gradient method is $10^{-6}$. The results show that heat flux is estimated well (Figure 6(a)). Thus, the heat loss from the insulation to environment is almost zero, but the amount of heat stored in the first layer of insulation is remarkable and almost $37 \%$ of the heat flux generated by the heater. In the next step, value of the heat transferred from heater to metal plate is calculated. In indirect estimation, the first value of heat transfer from metal plate to fluid is estimated and then convective heat coefficient is calculated by using Newton's law of cooling. Pulse sensitivity coefficient equation for

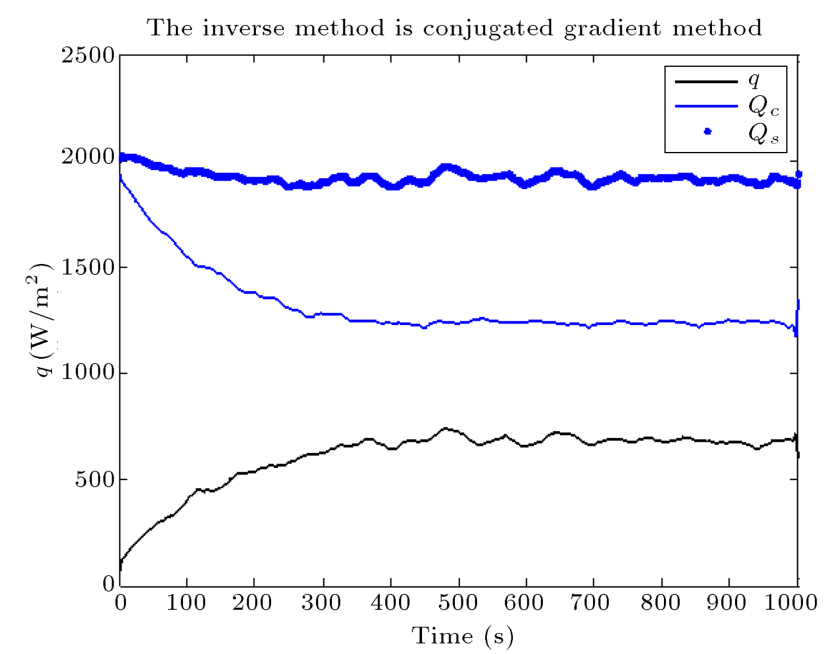

(a)

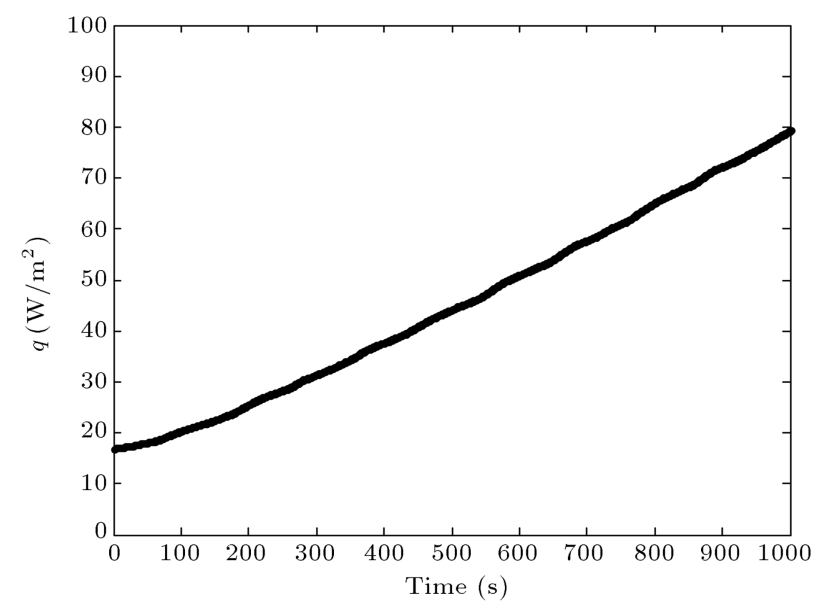

(b)

Figure 6. (a) Estimation of heat loss and heat flux subjected to metal plate, and (b) estimation of radiation heat flux transferred from metal plate to fluid by using Conjugate Gradient Method (CGM) in the third experiment. 
indirect estimation is obtained by taking the derivative of the heat Eq. (4) with respect to each heat flux, $q^{\prime}$, which yields:

$$
\begin{aligned}
& \frac{\partial^{2} X}{\partial y^{2}}=\frac{1}{\alpha} \frac{\partial X}{\partial t}, \\
& \left.\frac{\partial X}{\partial y}\right|_{y=0}=0, \\
& -\left.k \frac{\partial X}{\partial y}\right|_{y=E}=\left\{\left.\begin{array}{ll}
1 & x_{n}<x<x_{n+1} \\
0 & \text { otherwise }
\end{array} \quad X\right|_{t=0}=0 .\right.
\end{aligned}
$$

Sensitivity coefficient is linear and dependent on the unknown parameter. In all of the calculations, sensitivity coefficient is constant. Emissivity coefficient of metal plate surface is measured by IR radiation thermometer and its value is 0.85 . Convection heat flux and radiation heat flux are estimated by the mentioned method (Figure 6(b)). The negative sign for heat flux is the heat transfer from metal plate to the environment. The radiation heat flux is little. Figure $7(\mathrm{a})$ shows time history of convective heat transfer coefficient. Average value of time for $h$ is $6.06 \mathrm{w} / \mathrm{m}^{2} \mathrm{~K}$. The actual average free convection heat transfer coefficient is calculated by the relationships presented for the horizontal plate in [14] and its value is $5.8 \mathrm{w} / \mathrm{m}^{2} \mathrm{~K}$. The relative error for this estimation is $4.47 \%$. In the last step, convective heat transfer coefficient is estimated by direct estimation. In direct estimation, conjugate gradient method with adjoint equation is used. The error function $S$ in integral form is also defined as:

$$
S=\int_{t=0}^{t_{f}} \int_{x=0}^{L_{1}} \int_{y=0}^{E}[Y-T] \delta\left(x-x_{s}\right) \delta\left(y-y_{s}\right) d y d x d t
$$

where $Y$ is measured temperatures at sensor location, and $T$ is the estimated value at sensor location. In Eq. (31), $x_{s}$ and $y_{s}$ refer to the location of sensor and $\delta($.$) is the Dirac delta function. A Lagrange multiplier$ $\lambda(x, t)$ comes into picture in the minimization of the function in Eqs. (5) because the temperature $T(x, t ; h)$ appearing in such function needs to satisfy a constraint which is the solution of the direct problem. Such Lagrange multiplier, needed for the computation of the gradient equation (as will be apparent below), is obtained through the solution of a problem adjoint to the sensitivity problem given by Eq. (32).

In order to derive the adjoint problem, we write the following extended function:

$$
S(T, h)=\int_{t=0}^{t_{f}} \int_{x=0}^{L_{1}} \int_{y=0}^{E}\left[[Y-T]^{2} \delta\left(x-x_{s}\right) \delta\left(y-y_{s}\right)\right.
$$

$$
\left.-\lambda(x, y, t)\left(\frac{\partial^{2} T}{\partial x^{2}}+\frac{\partial^{2} T}{\partial y^{2}}-\frac{1}{\alpha} \frac{\partial T}{\partial t}\right)\right] d y d x d t .
$$

An expression for the variation $\Delta S(h)$ of the function $S(h)$ can be developed by perturbing $T(x, t)$ by $\Delta T(x, t)$ in Eq. (5). We note that $\Delta S(h)$ is the directional derivative of $S(h)$ in the direction of the perturbation $\Delta h=\left[\Delta h_{1}, \cdots, \Delta h_{n}\right]$. Then, by replacing $T(x, t)$ with $[T(x . t)+\Delta T(x, t)]$ and $S(h)$ with $[S(h)+\Delta S(h)]$ in Eq. (5), subtracting the original Eq. (5) from the resultant expression, and neglecting second-order terms, we find:

$$
\begin{aligned}
\Delta S(T, q) & \\
= & \int_{t=0}^{t_{f}} \int_{x=0}^{L_{1}} \int_{y=0}^{E}\left[2[T-Y] \delta\left(x-x_{s}\right) \delta\left(y-y_{s}\right) \Delta T(x, t)\right.
\end{aligned}
$$

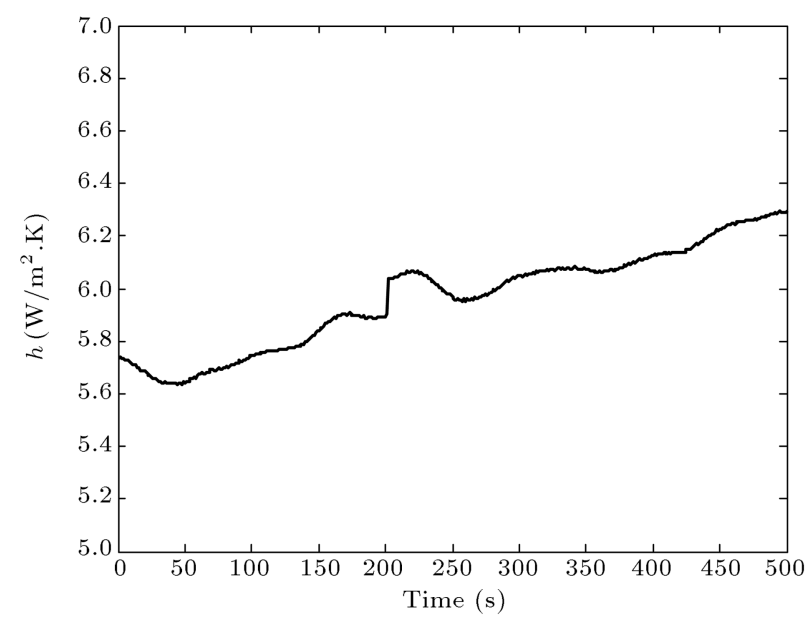

(a)

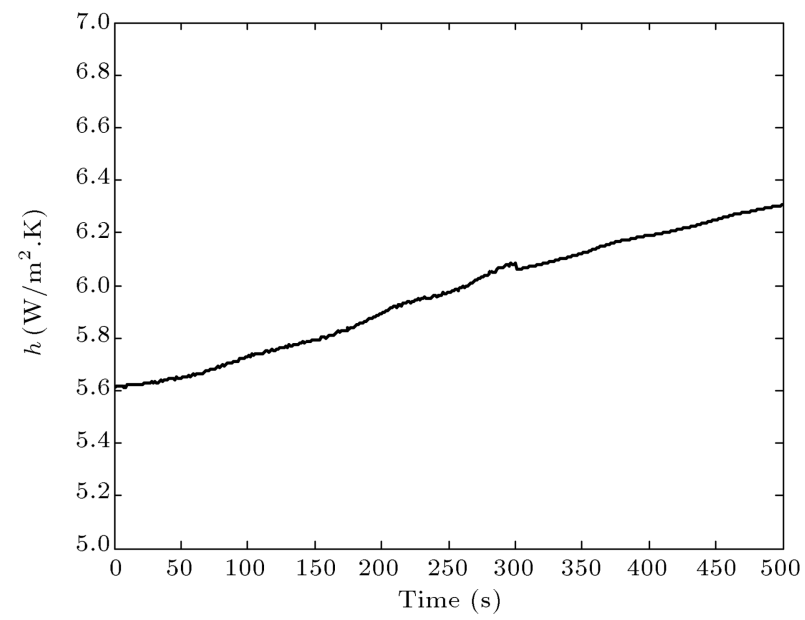

(b)

Figure 7. (a) Indirect estimation time history of convective heat transfer coefficient, and (b) direct estimation time history of convective heat transfer coefficient in the third experiment. 


$$
\left.+\lambda(x, y, t)\left(\frac{\partial^{2}(\Delta T)}{\partial x^{2}}+\frac{\partial^{2}(\Delta T)}{\partial y^{2}}-\frac{1}{\alpha} \frac{\partial(\Delta T)}{\partial t}\right)\right]
$$

$d y d x d t$

The second integral term on the right-hand side of this equation is simplified by integration of parts and by utilizing the boundary and initial conditions of the sensitivity problem. After some manipulation, the adjoint differential equations are obtained as:

$$
\begin{aligned}
& \lambda_{x x}+\lambda_{y y}+2 \sum_{i=1}^{N_{s}}(T-Y) \delta\left(x-x_{i}\right) \delta\left(y-y_{i}\right)=-\lambda_{t} / \alpha, \\
& \lambda_{x \mid x=0}=\lambda_{x \mid x=l}=0 \quad \text { and } \quad \lambda\left(t_{f}\right)=0, \\
& \lambda_{y \mid y=0}=0 \quad \text { and } \\
& -k \lambda_{y \mid y=E}=\left(h+4 \varepsilon \sigma T^{3}(x, E, t)\right) \lambda \\
& d f=d L=\frac{\partial L}{\partial h} d h \Rightarrow \nabla f \\
& =-\lambda(x, E, t)\left(T(x, E, t)-T_{\infty}\right) / k .
\end{aligned}
$$

Note that in the adjoint problem, the condition $\left(\lambda\left(t_{f}\right)=0\right)$ is the value of the function $\lambda(x, t)$ at the final time $t=t_{f}$. Thus, this equation must be solved backward. The gradient of the objective function is obtained from the adjoint equations. Figure $7(\mathrm{~b})$ shows time-varying convective heat transfer coefficient. Its average value of time is $5.76 \mathrm{~W} / \mathrm{m}^{2} \mathrm{~K}$. The relative error for direct estimation is $1 \%$.

In the review and analysis of three experiments, it was found that if the mathematical model is consistent with the experimental model, inverse technique has good accuracy and high reliability and is needed for low equipment. With this investigation, it is approved that the inverse whole-domain method is more accurate. This study is a starting point for using inverse methods in experimental investigation phenomena, such as boiling in channel, impingement jet, etc.

\section{Conclusion}

This study investigated the reliability and accuracy of the inverse heat transfer method in experimental problems. Heat flux was estimated with good accuracy by using inverse methods (CGM and SFSM) in the first and second experiments. In the third experiment, heat flux value transferred from the heater to insulation was estimated by using the inverse method, CGM. Free convection heat transfer coefficient is estimated by the two methods with good accuracy. As seen in the results, direct estimation is more accurate than indirect estimation. By the performed three experiments, it is found that precision of the mathematical model for the problem, correctness of its boundary conditions, and appropriateness of the boundary conditions and the experimental model are essential. In experimental state, it is difficult to find an appropriate model compared to when the measurement data are obtained from the numerical simulations. The results show that the desired parameters can estimate with good accuracy by using simple and inexpensive equipment and using standard inverse methods. The inverse method can be a practical tool in experiment.

\section{Nomenclature}

$A$

$D$

Vector of unity matrices

Bias error

Plate thickness

$h$

Heat transfer coefficient $\left(\mathrm{W} / \mathrm{m}^{2} \mathrm{~K}\right)$

$k$ Thermal conductivity (W/m.K)

L Plate length

$M \quad$ Time index

$N$

Number of discrete measurements

$N p \quad$ Number of unknown parameters

Ns Number of sensors

$q \quad$ Heat flux vector $\left(\mathrm{W} / \mathrm{m}^{2}\right)$

$Q_{s} \quad$ Heat flux generated by heater $\left(\mathrm{W} / \mathrm{m}^{2}\right)$

$r \quad$ Number of future time steps

RMS Root Mean Square error

$S \quad$ Sum of squares $\left(\mathrm{K}^{2}\right)$

$D \quad$ Conjugate direction

$T \quad$ Vector of calculated temperatures

$V \quad$ Variance error

$W \quad$ Slot width

$X \quad$ Sensitivity coefficient matrix $(\mathrm{K} / \mathrm{W})$

$x, y \quad$ Space coordinates

$Y \quad$ Measured temperature

$Z \quad \mathrm{XA}$

\section{Greek symbols}

$\alpha \quad$ Thermal diffusivity $\left(\mathrm{m}^{2} / \mathrm{s}\right)$

$\sigma \quad$ Standard deviation of noise

$\beta \quad$ Search step size

$\varepsilon \quad$ Surface emissivity coefficient

$\gamma \quad$ Conjugate coefficient

\section{Subscripts}

$0 \quad$ Initial state

$J \quad$ Position index

Surf Surface 
$\begin{array}{ll}\text { Ins } & \text { Insulation } \\ \infty & \text { Ambient condition }\end{array}$

\section{References}

1. Tikhonov, A.N. and Arsenin, V.Y., Solution of IllPosed Problems, Winston and Sons, Washington. D.C. (1977).

2. Chin-Ru, S., Cha'o-Kuang, C., Wei-Long, L. and Hsin-Yi, L. "Estimation for inner surface geometry of furnace wall using inverse process combined with grey prediction model", International Journal of Heat and Mass Transfer, 52(15), pp. 3595-3605 (2009).

3. Beck, J.V. "Transient determination of thermal properties", Nuclear Engineering and Design, 3, pp. 373381 (1966).

4. Jurkowski, T. and Jarny, Y. "Simultaneous identification of thermal conductivity and thermal contact resistance without internal temperature measurements", Institution of Chemical Engineers Symposium Series, 2(129), pp. 1205-1211 (1992).

5. Garnier, B., Delaunay, D. and Beck, J.V. "Estimation of thermal properties of composite materials without instrumentation inside the samples", International Journal of Thermo-Physics, 13(6), pp. 1097-1111 (1992).

6. Hanak, J.P. "Experimental verification of optimal experimental designs for the estimation of thermal properties of composite materials", M.S. Thesis, Department of Mechanical Engineering, Virginia Polytechnic Institute and State University, Blacksburg, VA, USA (1995).

7. Taler, J. "Determination of local heat transfer coefficient from the solution of the inverse heat conduction problem", Forsch. Ingenieurwes., 71, pp. 69-78 (2007).

8. Dong, C.F., Sunb, F.Y. and Mengc, B.Q. "A method of fundamental solutions for inverse heat conduction problems in an anisotropic medium", Engineering Analysis with Boundary Elements, 31, pp. 75-82 (2007).

9. Beck, J.V., Blackwell, B. and Clair, S.R., Inverse Heat Conduction: Ill-Posed Problems, Wiley, New York (1988).

10. Woodbury, K.A., Inverse Engineering Handbook, CRC Press (2003).
11. Ozisik, M.N. and Orlande, H.R.B., Inverse Heat Transfer Fundamentals and Applications, Taylor \& Francis (2000).

12. Kowsary, F. and Farahani, S.D. "The smoothing of temperature data using the mollification method in heat flux estimating", Numerical Heat Transfer: Part A, 58, pp. 227-246 (2010).

13. Woodbury, K.A. and Beck, J.V. "Estimation metrics and optimal regularization in a Tikhonov digital filter for the inverse heat conduction problem", International Journal of Heat and Mass Transfer, 62, pp. 3139 (2013).

14. Incropera, F.P., Dewitt, D.P., Bergman, T.L. and Lavine, A.S., Introduction to Heat Transfer, Willey Press (2006).

\section{Biographies}

Somayeh Davoodabadi Farahani is a $\mathrm{PhD}$ student of Heat Transfer at University of Tehran, Iran. Her research areas of interest include the use of numerical and analytical solution methods of the heat transfer problems, direct simulation of thermal systems, solution of inverse heat transfer problems, optimization of thermal systems, nanofluid, and nanoscale heat transfer.

Ali-Reza Najafi is an MS student of Heat Transfer at University of Tehran, Iran. His research areas of interest include the use of numerical and analytical solution methods of the heat transfer problems.

Farshad Kowsary is Professor of Heat Transfer at University of Tehran, Iran. His research interests are in the area of inverse heat transfer with a focus on inverse radiation and conduction. He has a sizable number of papers on these subjects in reputable heat transfer journals. He is a major reviewer of the Journal of Quantitative Spectroscopy and Radiative Transfer and Heat and Mass Transfer.

Mehdi Ashjaee is Professor of Heat Transfer at University of Tehran, Iran. His research interests are in the area of inverse heat transfer with a focus on experimental heat transfer by using interferometry. He has a sizable number of papers on these subjects in reputable heat transfer journals. 